\title{
Hospital-based Population of Elderly Cancer Cases in Northeastern Thailand
}

\author{
K Wirasorn ${ }^{1}$, K Suwanrungruang ${ }^{2}$, A Sookprasert ${ }^{1}$, P Limpawattana ${ }^{3}$, W \\ Sirithanaphol $^{4}$, J Chindaprasirt ${ }^{1 *}$
}

\begin{abstract}
Background: The proportion of aged Thais ( $\geq 65$ years old) is expected to be $30 \%$ by 2030 , leading to an increased number of elderly cancer cases. Older individuals have distinct patterns of cancer and treatment needs. We therefore conducted the present study of new cancer cases and trends to get a perspective on the elderly cancer situation in Northeast Thailand. Materials and Methods: All new elderly cancer cases ( $\geq 65$ years) registered in the hospital-based cancer registry at the Faculty of Medicine, Srinagarind Hospital, Khon Kaen University during 1993-2012 were included in the study. Results: Elderly patients accounted for $31.6 \%$ of all cancer patients and new cancer cases in the older age group increased $46 \%$ from the first to second decades. The absolute number of oldest old (80+ years) doubled. The top three cancers in males were liver and bile duct, lung, and colorectal. In females, the three most common cancers were liver and bile duct, oral cavity, and cervix. Cancers with the highest percentages of increase were thyroid, prostate, and colorectal. Conclusions: Elderly cancer cases are increasing. Treatment modalities and palliative care for older populations are urgently needed.
\end{abstract}

Keywords: Geriatric oncology - aging - elderly cancer case prevalence - North-east Thailand

Asian Pac J Cancer Prev, 17 (2), 767-770

\section{Introduction}

A rapid decline in fertility and improved life expectancy result in the dramatic increase in older adults, both absolute numbers and as a ratio in Thailand (Knodel et al., 2011). The proportion of older individuals will increase from 13 percent in 2010 to 30 percent by 2030 (Office, 2014).

Aging is a significant risk factor for developing cancer (Lichtman et al., 2014). As people get older, more will suffer from cancer. More than $60 \%$ of cancers are diagnosed in those age more than 65 years old (Yancik, 1997). Since Thailand is moving toward an ageing society, the number of cancer diagnoses in elderly patients is expected to increase.

Dealing with elderly cancer is a challenge for oncologists. Not only the limitation in functional status, but also the problems with co-morbidity, frailty, polypharmacy, and treatment toxicity (Lichtman et al., 2014; Maggiore et al., 2014). Both the disease and its treatment affect overall life expectancy, quality of life, and burden for the caregivers (Chindaprasirt et al., 2014).

Therefore, we conducted this overview of elderly cancer data derived from Srinagarind Hospital cancer registry to get a perspective of the trends of cancer type and its impact in Northeastern Thailand.

\section{Materials and Methods}

\section{Population and registration procedure}

Case definitions: All new cancer cases aged $\geq 65$ years registered in Srinagarind Hospital between January 1st, 1993 and December $31^{\text {st }}, 2012$ were included in this study. This study is officially approved by the Khon Kaen University Ethics Committee for Human Research based on the Declaration of Helsinki and the ICH Good Clinical Practice Guidelines with HE571238 of reference number.

Sources of data: Srinagarind hospital, Khon Kaen University (a thousand-bed university hospital), Khon Kaen, Thailand, is situated in the center of NE Thailand and accepts all referred cases from other health care centers in the region. Our data were obtained from the Khon Kaen Cancer Registry, which has recorded data of all cancer cases treated in this hospital since 1987. All data were verified, checked for coding duplication and entered in to the CANREG software (Version 4, available from http://www.iacr.com.fr/canreg4.htm). The data normally collected from each cancer patient included age, sex, date of birth, date of diagnosis, method of diagnosis, primary site of cancer, extension or metastasis, histology of cancer, date of last visit, vital status at last follow-up, and other necessary information. Deaths were not recorded but these data can be obtained from the civil registration database, 
$K$ Wirasorn et al

if they are required. Only relevant data were presented in this study.

\section{Statistical methods}

The data were analyzed using Poisson regression to compare the trends in the first decade (1993-2002) and the second decade (2003-2012).

\section{Results}

During 1993-2012, a total of 73,218 new cancer cases were diagnosed in Srinagarind Hospital and elderly patients ( $\geq 65$ years) accounted for $31.6 \%$ of all cancer patients. The number of older patients increased from the first to the second decade, but the mean age was comparable; 71.2 vs 71.9 years (Table 1). From from the first to the second, new elderly cancer cases increased by $46 \%$. The absolute number of patients increased in all age groups but the number of the oldest old ( $\geq 80$ years) doubled. The number of cancer cases had been rather stable from 1993-2005, but increased gradually since then (Figure 1). Male patients outnumbered female patients in all age groups except those aged more than 80 .

Table 1 . Gender and age distribution of cancer cases in patients age $\geq 65$ years diagnosed during 1993-2012 (the first decade) and 2003-2012 (the second decade).

The top three cancers for overall population over the age of 65 years were liver and bile duct, head and neck, and lung cancer as shown in Figure 2. Most of the patients

Table 1. Gender and Age Distribution of Cancer Cases in Patients age $\geq 65$ Years Diagnosed During 1993-2012 (First Decade) and 2003-2012 (Second Decade)

\begin{tabular}{lcccc}
\hline Characteristics & \multicolumn{2}{c}{$1993-2002$} & \multicolumn{2}{c}{$2003-2012$} \\
\cline { 2 - 5 } & Number & $\%$ & Number & $\%$ \\
\hline Gender & & & & \\
Male & 5,598 & 59.5 & 7,831 & 57.5 \\
Female & 3,809 & 40.5 & 5,903 & 43 \\
Age (year) & & & & \\
$65-69$ & 4,412 & 46.9 & 5,543 & 41.1 \\
$70-74$ & 2,777 & 29.5 & 4,138 & 30.1 \\
$75-79$ & 1,416 & 15.1 & 2,456 & 17.9 \\
$80-84$ & 547 & 5.81 & 1,108 & 8.1 \\
$\geq 85$ & 255 & 2.71 & 389 & 2.8 \\
Mean(SD) & $71.2(5.4)$ & $71.9(5.5)$ \\
\hline
\end{tabular}

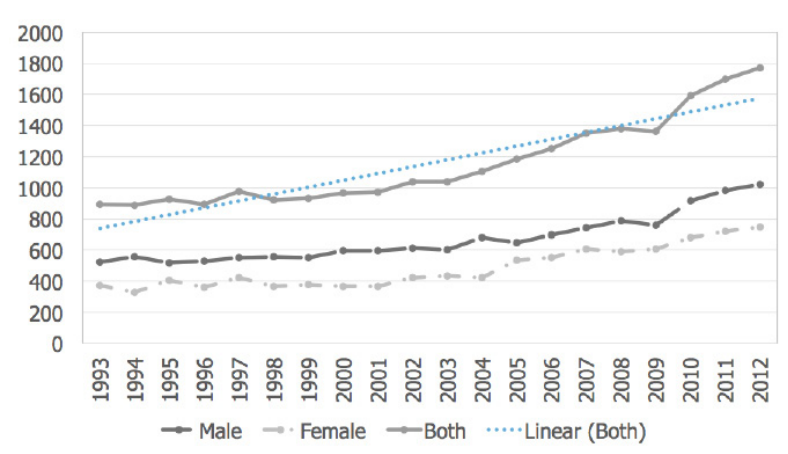

Figure 1. Overall Numbers of New Elderly Cancer Cases During 1993-2012 age 65-69 years. In male patients, the top five cancers were liver and bile duct, lung, colorectal, oral cavity, and prostate cancer (Figure 3A). In female patients, the top five cancers were liver and bile duct, oral cavity, cervix uteri, colorectal, and lung cancer (Figure 3B).

In males, cancer sites with the highest percentage of increase between 1993 through 2012 were prostate $(123 \%)$, colorectal $(99 \%)$, non-Hodgkin lymphoma $(67 \%)$, and bladder (49\%). The number of oral cavity cancer cases decreased $10 \%$.

In females, cancer sites with the highest percentage of increase between 1993 through 2012 were thyroid (186\%), colorectal $(134 \%)$, lung $(92 \%)$, breast $(85 \%)$, and ovary $(85 \%)$. Cervical cancer cases reduced by $6 \%$ from the first to the second.

Liver and bile duct cancer was the most common cancer in both males and females. It has outnumbered other cancers and showed an accelerating increase during the last five years (Figure 3). Most of the patients aged 65-

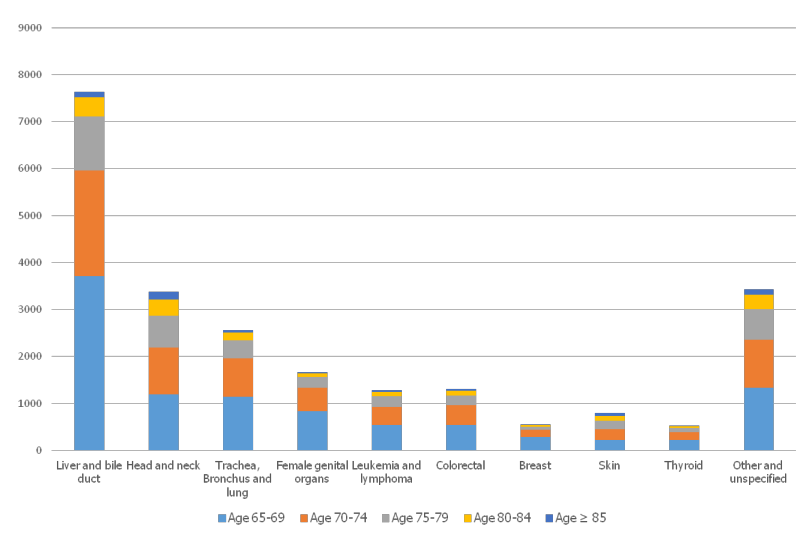

Figure 2. New Cancer Cases According to Primary Site and Age Group

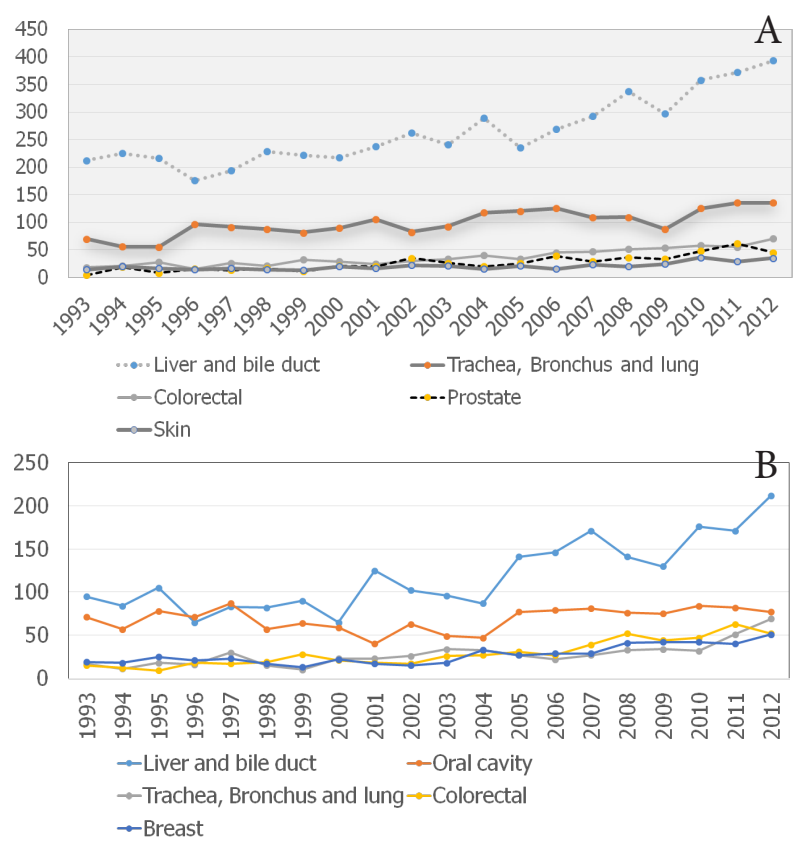

Figure 3. A) Numbers of Cases of the Leading Cancers in Males During 1993-2012; B) Numbers of Cases of the Leading Cancers in Females During 1993-2012 
Colorectal, male
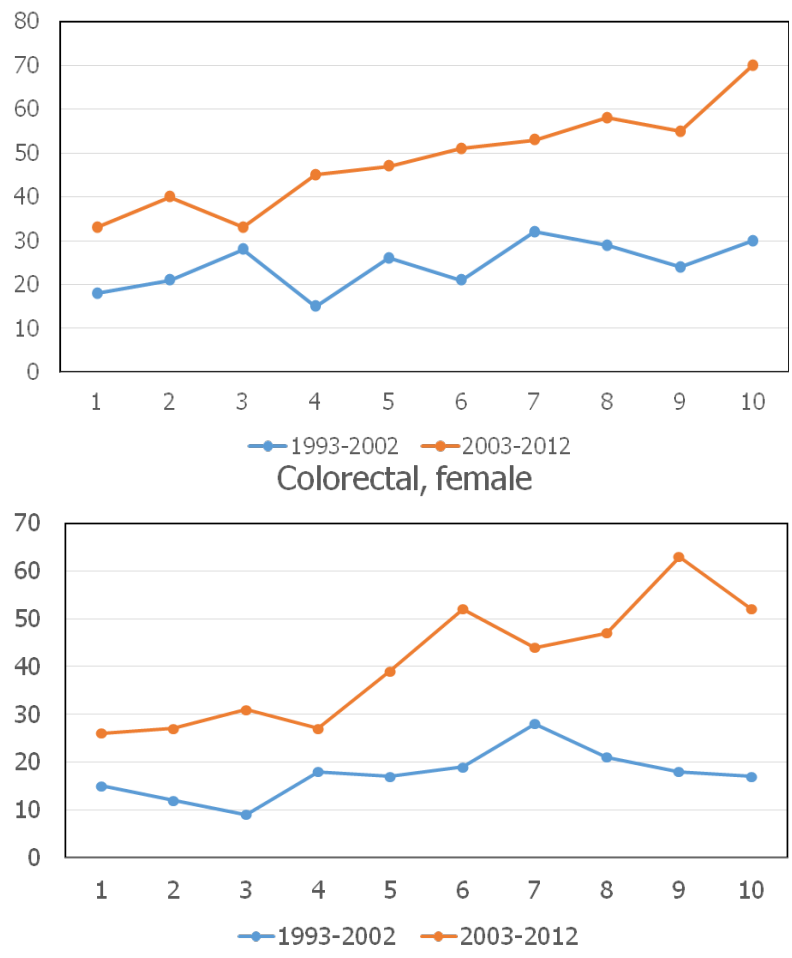

Figure 4. Numbers of Colorectal Cancer Cases in the First and Second Decade

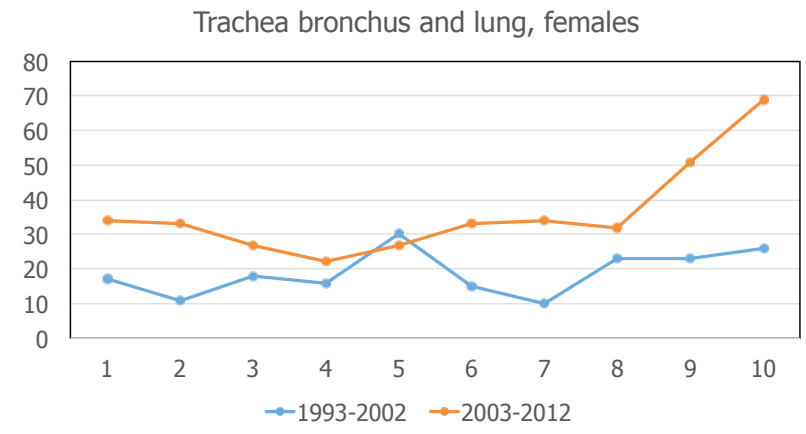

Figure 5. Numbers of Lung Cancer Cases in Females in the First and Second Decade

69 years old. In 1996, there were 241 cases and the number reached 605 cases in the year 2012. From from the first to the second decade, new liver and bile duct cancer cases have doubled in females and increased for $40 \%$ in males.

Colorectal cancer was the third and the fourth most common cancer in males and females, respectively. New patients increased substantially in both sexes and rose to nearly four-fold from 33 cases in 1993 to 122 cases in 2012 (Figure 4).

Lung cancer was the second most common cancer in males and the fifth in females. The figure of new cases was rather constant during the two decades for male patients, approximately 100 cases per year. In females, however, the total number markedly rose especially in the last few years (Figure 5).

Interestingly, thyroid cancer figures considerably increased in older women, with only 93 cases in the first decade to 266 cases in the second. Likewise, breast and ovarian cancer numbers also gradually increased in the last 10 years. The figures for both cancers nearly doubled from the first to the second decade.

\section{Discussion}

We found that the number of new elderly cancer cases increased substantially especially in the last five years. Liver and bile duct cancer was the most common in older men and women from Northeastern Thailand. Interestingly, prostate, thyroid, and colorectal cancer numbers had the highest percentage of increase of new cases from the first to the second decade.

The number of elderly cases rose in the last few years. The finding is consistent with other hospital-based registry in Thailand and data from the US and the UK (Attasara et al., 2014; Surveillance, 2015; UK, 2015). The increase of overall cancers in elderly could be due to an expanded population size, demographic shift toward aging society in Thailand, better diagnostic tools, and easier access to healthcare system from the policy of universal coverage since 2001.

Liver and bile duct cancer have always been the burden of this region of the world. Despite the effort in promoting healthier and safer lifestyle to prevent opisthrochis viverrini infestation, new cases are still sky high. The same trend as the all age-group population was reported earlier (Wirasorn et al., 2014). Surgery is the most effective therapy for the disease (Friman, 2011) however, most of the older patients may not be able to tolerate major surgery like hepatectomy or wedge resectio. They could not tolerate chemotherapy either (Sookprasert et al.,2012). The mortality for older patients with cholangiocarcinoma is likely to be high and it is an urgent issue for healthcare workers in this region.

Although prostate cancer cases have doubled from from the first to the second decade, the total number of cases was fewer compared with the data from UK or Norway (Syse et al., 2012; UK, 2015). The number of thyroid cases surprisingly rose in older women in the second decade. We do not have the mortality data, but we assume that it must be low, inferred from the data from other parts of the world (Pellegriti et al., 2013). The experience of overdiagnosis of thyroid cancer from South Korea made us to consider more about screening or even operating in thyroid cancer especially in older age group (Ahn et al., 2014).

Our data could not refer to community incidences which was the major limitation of this study. Since we reported only hospital-based cases and there was no data about mortality rate, we could not conclude entirely the real burden of elderly cancer.

In conclusion, elderly cancer cases have been increasing and liver and bile duct cancer is still the burden in this part of the world. Treatment modalities and palliative care for older populations are urgently needed.

\section{Acknowledgements}

The Cancer Research Group, Khon Kaen University, Thailand. 


\section{$K$ Wirasorn et al}

\section{References}

Ahn HS, Kim HJ, Welch HG (2014). Korea's thyroid-cancer "epidemic"--screening and overdiagnosis. $N$ Engl J Med, 371, 1765-7.

Attasara P, Buasom R, Kookiang J, et al (2014). Hospital-based cancer registry annual report 2012, Bangkok, Eastern Printing Public Company Limited PCL.157.

Chindaprasirt J, Limpawattana P, Pakkaratho P, et al (2014). Burdens among caregivers of older adults with advanced cancer and risk factors. Asian Pac J Cancer Prev, 15, 1643-8.

Friman S (2011). Cholangiocarcinoma--current treatment options. Scand J Surg, 100, 30-4.

Knodel J, Chayovan N, Prachuabmoh V (2011). Impact of population change on well-being of elderly in Thailand. In 'Impact of Demographic Change in Thailand', Eds UNFPA, Bangkok, 35-63

Lichtman SM, Hurria A, Jacobsen PB (2014). Geriatric oncology: an overview. J Clin Oncol, 32, 2521-2.

Maggiore RJ, Dale W, Gross CP, et al (2014). Polypharmacy and potentially inappropriate medication use in older adults with cancer undergoing chemotherapy: effect on chemotherapyrelated toxicity and hospitalization during treatment. $\mathrm{J} \mathrm{Am}$ Geriatr Soc, 62, 1505-12.

Pellegriti G, Frasca F, Regalbuto C, et al (2013). Worldwide increasing incidence of thyroid cancer: update on epidemiology and risk factors. J Cancer Epidemiol, 2013, 965212.

Sookprasert A, Chindaprasirt J, Wirasorn K (2012). Systemic therapy for locally advanced and metastatic cholangiocarcinoma. Asian Pac J Cancer Prev, 13, 3-6.

Surveillance E, End Results (SEER) Program. 2015. Available: http://seer.cancer.gov/faststats/selections.php?series=age [Accessed 20 September 2015].

Syse A, Veenstra M, Aagnes B, et al (2012). Cancer incidence, prevalence and survival in an aging Norwegian population. Norsk Epidemiologi, 22, 109-20.

UK CR. (2015). Available: http://www.cancerresearchuk.org/ health-professional/cancer-statistics/incidence/age\#headingZero [Accessed 20 September 2015].

Wirasorn K, Suwanrungruag K, Wiangnon S, et al (2014). Numbers of new cases and trends of cancer 1993-2012: Srinagarind hospital based population, Khon Kaen, NorthEast Thailand. Asian Pac J Cancer Prev, 15, 8423-7.

Yancik R (1997). Cancer burden in the aged: an epidemiologic and demographic overview. Cancer, 80, 1273-83. 